\title{
SUMMABILITY INTEGRALS
}

\author{
GEORGE BRAUER
}

\begin{abstract}
An integral on the set of natural numbers $N$ is defined. If $E$ is a subset of $N$ such that the characteristic function $\chi_{E}$ of $E$ is evaluated to some number $\sigma$ by a regular nonnegative summation matrix $A$, then $\mu_{A}(E)$ is defined to be $\sigma ; \mu_{A}$ defines a finitely additive measure on $N$. If $s$ is a sequence which can be written as a linear combination of characteristic functions $\sum_{i=1}^{n} a_{i} \chi_{E_{i}}$, where each sequence $\chi_{E_{i}}$ is evaluated by $A$, then $\int_{N} s d \mu_{A}$ is defined to be $\sum a_{i} \mu_{A}\left(E_{i}\right)$. Finally the definition of the integral is naturally extended to $L(A)$, the class of sequences which can be approximated by linear combinations of characteristic functions [2, pp. 85-88]. It is shown that if $A$ and $B$ are two nonnegative regular matrices such that the convergence field of $A$ includes that of $B$, then $L(A)$ includes $L(B)$ provided $B$ is normal. Finally for a nonnegative regular matrix $A=\left(a_{n k}\right)$, the spaces $L^{p}(A)$ spanned by sequences such that $\left\{\sum_{k=0}^{\infty} a_{n k}\left|s_{k}\right|^{p}\right\}$ is bounded and $\lim \sum_{k=0}^{\infty} a_{n k} s_{k}^{p}$ exists are studied. It is shown that if $p$ is greater than one, then the sequences in $L^{p^{\prime}}(A)$ give rise to a set of bounded linear functionals on $L^{p}(A)$ which are weak star dense in the dual of $L^{p}(A)$.
\end{abstract}

Let $A=\left(a_{n k}\right)$ be a nonnegative regular summation matrix and let $\Sigma=\Sigma_{A}$ denote the class of subsets $E$ of the natural numbers $N$ such that

$$
\lim _{n \rightarrow \infty} \sum_{k \in E} a_{n k}=\mu_{A}(E)
$$

exists, that is, $\Sigma$ denotes the class of sets $E$ whose characteristic functions $\chi_{E}$ are $A$-summable; thus $\mu_{A}$ is a finitely additive measure on $N$ (the characteristic function of $E$ is the sequence which is one when $n$ is in $E$ and zero when $n$ is not in $E)$. Let $S\left(\Sigma_{A}\right)$ denote the set of finite linear combinations $\sum a_{i} \chi_{E_{i}}$, where the $a_{i}$ are scalars and the sets $E_{i}$ are in $\Sigma$. Each sequence $s$ in $S(\Sigma)$ lies in the convergence field, $W(A)$, of $A$; if we write

$$
\int_{N} s d \mu_{A}=\sum_{i=1}^{n} a_{i} \mu_{E_{i}}(A)
$$

for each sequence $s=\Sigma a_{i} \chi_{E_{i}}$ in $S(\Sigma), E_{i} \in \Sigma$, then

$$
\int_{N} s d \mu_{A}=\lim _{n \rightarrow \infty} \sum_{k=0}^{\infty} a_{n k} s_{k} .
$$

Edwards and Wayment [1, p. 88] define the summability integral as follows:

Presented to the Society, November 3, 1973 under the title Summability integration; received by the editors September 29, 1975.

AMS (MOS) subject classifications (1970). Primary 40C05; Secondary 28A25.

Key words and phrases. Linear combinations of characteristic functions, linear functionals, dual, weak star dense. 
the norm of a sequence $s$ in $S(\Sigma)$ is defined by

$$
\|s\|=\|s\|_{A}=\lim \sup _{n} \sum_{k=0}^{\infty} a_{n k}\left|s_{k}\right| .
$$

$L(A)$ denotes the closure of $S\left(\Sigma_{A}\right)$ in the norm (1). If $s \in L(A)$, then for each $\varepsilon>0$ there exists a sequence $s_{\varepsilon}$ in $S(\Sigma)$ such that $\left\|s-s_{\varepsilon}\right\| \leqslant \varepsilon$; we define

$$
\int s d \mu_{A}=\lim _{\varepsilon \rightarrow 0} \int s_{\varepsilon} d \mu_{A} .
$$

Clearly $\int s d \mu_{A}$ defined above is independent of the choice of $s_{\varepsilon}$. We norm $L(A)$ by

$$
\|s\|=\|s\|_{A}=\lim \sup _{n} \sum_{k=0}^{\infty} a_{n k}\left|s_{k}\right| .
$$

Two sequences $s$ and $t$ are identified in $L(A)$ whenever $\|s-t\|=0$. Formula (1') extends the definition of the norm from $S(\Sigma)$ to $L(A)$.

For each $E \subseteq N$ the symbol $\bar{\mu}_{A}(E)$ will denote $\lim \sup \sum_{k \in E}\left|a_{n k}\right| ; \mu_{A}$ is an outer measure, and $\bar{\mu}_{A}(E)=\left\|\chi_{E}\right\|$ if $\chi_{E} \in L(A)$.

Edwards and Wayment [1, p. 92] ask whether $L(A) \supseteq L(B)$ implies $W(A)$ $\supseteq W(B)$, where $W(A)$ denotes the convergence field of the summation matrix $A$. We will exhibit two nonnegative regular matrices $A$ and $B$ such that $L(A) \supseteq L(B)$ while $W(A) \unrhd W(B)$. Let $A$ be given by the equations

$$
\begin{aligned}
a_{n, n}=1, \quad a_{n, k}=0 & \text { when } k \neq n, n \text { even, } \\
a_{n, n-1}=1, & a_{n, k}=0
\end{aligned}
$$

and let $B$ be the Nörlund matrix given by the equations

$$
\begin{aligned}
& b_{00}=1, \quad b_{0 k}=0, \quad k \neq 0, \\
& b_{n, n}=b_{n, n-1}=1 / 2, \\
& b_{n, k}=0, \quad k \neq n, k \neq n-1, n \geqslant 1 .
\end{aligned}
$$

The only sequences $s$ in $L(B)$ are those for which both subsequences $\left\{s_{2 n}\right\}$ and $\left\{s_{2 n+1}\right\}$ converge, while $L(A)$ contains all sequences $t$ such that $\left\{t_{2 n}\right\}$ converges; hence $L(A) \supseteq L(B)$. However, $A$ evaluates only those sequences $s$ such that the subsequence $\left\{s_{2 n}\right\}$ converges, while $B$ evaluates all sequences $s$ such that $\left\{s_{n}+s_{n+1}\right\}$ converges. Hence $W(A) \nsupseteq W(B)$.

In the reverse direction we have

THEOREM. If $A$ and $B$ are nonnegative regular matrices, $B$ is normal and $W(A) \supseteq W(B)$, then $L(A) \supseteq L(B)$.

A normal matrix is a triangular matrix with all diagonal elements nonzero.

Proof. Suppose that $s$ is a sequence in $L(B)$. Then for each $\varepsilon>0$ there exist sets $E_{i} \in \Sigma_{B}(i=1,2, \ldots, r)$ and scalars $a_{i}$ such that $\left\|s-\sum_{i=1}^{r} a_{i} \chi_{E_{i}}\right\|_{B}$ $\leqslant \varepsilon$. Since each sequence $\chi_{E_{i}}$ is evaluated by $B$, it is evaluated by $A$, that is, $E_{i} \in \Sigma_{A}$ for each $i$. Since $B$ is normal, $B^{-1}$ exists. Our hypothesis $W(A) \supseteq$ $W(B)$ implies that the matrix $A B^{-1}$ is regular. Thus $A B^{-1}$ represents a bounded operator on the Banach space $m_{0}$ of bounded sequences, normed by

$$
|||s| \|=\lim \sup \left|s_{n}\right|, \quad s \in m_{0} .
$$


(Sequences differing by a null sequence are identified in $m_{0}$.) Let $u$ $=\left|B^{-1}\left(s-\sum_{i=1}^{r} A_{i} \chi_{E_{i}}\right)\right|$, that is,

$$
u_{n}=\left|\left\{B^{-1}\left(s-\sum_{i=1}^{r} a_{i} \chi_{E_{i}}\right)\right\}{ }_{n}\right|, \quad n=0,1,2, \ldots
$$

We have $\|B u\|\|=\| u \|_{B} \leqslant \varepsilon$, and consequently $\|A u\|\|\leqslant\| A B^{-1} \| \mid \varepsilon$, where $\left\|A B^{-1}\right\| \mid$ denotes the norm of the operator on $m_{0}$ represented by the matrix $A B^{-1}$. (Again, since $A B^{-1}$ is regular, $\left\|A B^{-1}\right\| \mid<\infty$.) But

$$
\|\mid\| u\|\|=\left\|s-\sum a_{i} \chi_{E_{i}}\right\|_{A}
$$

and therefore this quantity is bounded by $\| A B^{-1}|| \varepsilon$. Since each set $E_{i}$ lies in $\Sigma_{A}, s \in L(A)$. This completes the proof.

We define, for a nonnegative regular summation matrix $A=\left(a_{n k}\right), p>1$, the space $L^{p}(A)$ consisting of linear combinations of sequences $s=\left\{s_{n}\right\}$ such that

and

$$
\|s\|_{p}=\lim \sup \left(\sum_{k=0}^{\infty} a_{n k}\left|s_{k}\right|^{p}\right)^{1 / p}<\infty,
$$

$$
\lim _{n \rightarrow \infty} \sum_{k=0}^{\infty} a_{n k} s_{k}^{p}
$$

exists. We note that $\left(8_{p}\right)$ defines a norm on $L^{p}(A)$. Edwards and Wayment [1, p. 92] raise the question whether the dual of $L^{p}(A)$ is $L^{p^{\prime}}(A), p^{\prime}=p /(p-1)$. In general each sequence $t \in L^{p^{\prime}}(A)$ gives rise to infinitely many bounded functionals on $L^{p}(A), p>1$. Suppose that $s \in L^{p}(A)$ and $t \in L^{p^{\prime}}(A)$. Then $s$ satisfies $\left(8_{p}\right)$ while $t$ satisfies $\left(8_{p^{\prime}}\right)$. The sequence $\left\{\sum_{k=0}^{\infty} a_{n k} s_{k} t_{k}\right\}$ is bounded; as a bounded function on the discrete space $N$ it has a continuous extension $\left\{\sum_{k=0}^{\infty} a_{n k} s_{k} t_{k}\right\}^{\beta}$ to $\beta N$, the Stone-Čech compactification of $N$. For a description of the Stone-Čech compactification, the reader is referred to $[2, \mathrm{pp}$. 82-89]. (Throughout the paper the superscript $\beta$ will indicate continuous extension to $\beta N$; for each point $\gamma \in \beta N-N$ we will indicate that the function $\left\{\sum_{k=j}^{\infty} a_{n k} s_{k} t_{k}\right\}^{\beta}$ has been evaluated at $\gamma$ by writing $\left\{\sum a_{n k} s_{k} t_{k}\right\}_{\gamma}^{\beta}$. If $s$ and $t$ are sequences such that $A$ fails to evaluate $s t$, then $t$ gives rise to bounded functionals

$$
L(t, \gamma) s=\left\{\sum_{k=0}^{\infty} a_{n k} s_{k} t_{k}\right\}_{\gamma}^{\beta}, \quad \gamma \in \beta N-N
$$

the functionals $L(t, \gamma)(s)$ take on different values as $\gamma$ ranges over the points of $\beta N-N$. als

THEOREM. If $A$ is a nonnegative regular matrix and $p>1$, then the function-

$$
L(t, \gamma)(s)=\left(\sum_{k=0}^{\infty} a_{n k} s_{k} t_{k}\right)_{\gamma}^{\beta}, \quad s \in L^{p}(A),
$$

where t ranges over $L^{p^{\prime}}(A)$ and $\gamma$ ranges over $\beta N-N$, are weak star dense in the dual of $L^{p}(A)$. 
Recall that the superscript $\beta$ in (10) indicates that the function $\left\{\sum a_{n k} s_{k} t_{k}\right\}$ has been continuously extended to $\beta N-N$; the subscript $\gamma$ indicates that the extended function has been evaluated at the point $\gamma$.

Proof. We first treat the case $p=2$. Let $s$ be a sequence in $L^{2}(A)$; then $\bar{s}=\left\{\bar{s}_{n}\right\}$ is in $L^{2}(A)$ and $\lim \sup \sum_{k=0}^{\infty} a_{n k} s_{k} \bar{s}_{k}=\|s\|_{2}^{2}$. There is a point $\gamma \in$ $\beta N-N$ such that $L(\bar{s}, \gamma)(s)=\|s\|_{2}^{2}$. Hence if $L(t, \gamma) s=0$ for all sequences $t \in L^{2}(A)$ and all points $\gamma \in \beta N-N$, then $\|s\|_{2}=0$; that is, for $p=2$, functionals of the form (10) are dense in the dual of $L^{p}(A)$.

For the other cases we need the following lemma whose proof is obvious.

Lemma. Let $0<\alpha<2 \pi, \alpha \neq \pi$ and let $\theta$ be a real number. If $\alpha$ is not a rational multiple of $2 \pi$, then for each $\varepsilon>0$ there exists an integer $m$ such that $|\theta-m \alpha|<\varepsilon(\bmod 2 \pi)$. If $\alpha$ is a rational multiple of $2 \pi$, then there exists an integer $m$ such that $|\theta-m \alpha|<\alpha / 2(\bmod 2 \pi)$.

We return to the proof of the theorem. Let $s$ be a sequence in $L^{p}(A)$; it is sufficient to show that if $s$ is a sequence in $L^{p}(A)$ and $\sum_{k=0}^{\infty} a_{n k} s_{k} t_{k}$ tends to zero for each sequence $t \in L^{p^{\prime}}(A)$, then $\|s\|_{p}=0$. Suppose that $\|s\|_{p}>0$. If $s \in L^{p}(A)$, then $s$ may be written $s=\sum_{i=1}^{r} s^{(i)}$ where each sequence $s^{(i)}$ satisfies $\left(8_{p}\right)$ and $\left(9_{p}\right)$. The sequences $\left(\bar{s}^{(i)}\right)^{p-1}(i=1,2, \ldots, r)$ satisfy $\left(8_{p^{\prime}}\right)$ and $\left(9_{p^{\prime}}\right)$ and hence the sequence $\bar{s}=\sum_{i=1}^{r}\left(\bar{s}^{(i)}\right)^{p-1}$ is in $L^{p^{\prime}}(A)$ as is each sequence

$$
t(m)=\left\{t_{k}(m)\right\}=\left\{\bar{s}_{k}^{p-1} \exp 2 m \pi i / p^{\prime}\right\}, \quad m=0,1, \ldots
$$

The case $2 \pi / p^{\prime}=\pi$ can occur only if $p=2$, and this case has been dealt with. Hence we may apply the lemma with $\alpha=2 \pi / p^{\prime} \neq \pi$ and $\theta$ as the angle between the vector $\bar{s}_{k}^{p-1}$ and the vector $u_{k}=\left|s_{k}\right|^{p-{ }_{s}} \bar{s}_{k}$ to conclude that if $p$ is irrational, then for each $\varepsilon>0$ there exists an integer $m_{k}$ such that the angle between the vector $t_{k}\left(m_{k}\right)$ and the vector $u_{k}$ is numerically less than $\varepsilon$ whenever $s_{k} \neq 0$, while if $p$ is rational, $p \neq 2$, then the angle between the vector $t_{k}\left(m_{k}\right)$ and the vector $u_{k}$ is numerically no greater than $\left|\pi-2 \pi / p^{\prime}\right|=\pi / p^{\prime}$ wherever $s_{k} \neq 0$. For such $m_{k}$,

$$
\operatorname{Re}\left|s_{k} t_{k}\left(m_{k}\right)\right| \geqslant\left|s_{k}\right|^{p} \cos \varepsilon
$$

if $p$ is irrational, while

$$
\operatorname{Re}\left|s_{k} t_{k}\left(m_{k}\right)\right| \geqslant\left|s_{k}\right|^{p} \cos \pi / p^{\prime}
$$

if $p$ is rational. Hence

$$
\lim \sup \sum_{k=0}^{\infty} a_{n k} s_{k} t_{k}\left(m_{k}\right) \geqslant\left\|s_{k}\right\|_{p}^{p} \cos \varepsilon
$$

if $p$ is irrational, while

$$
\lim \sup \sum_{k=0}^{\infty} a_{n k} s_{k} t_{k}\left(m_{k}\right) \geqslant\|s\|_{p}^{p} \cos \pi / p^{\prime}
$$

if $p$ is rational, $p \neq 2$. In either case if $\|s\|_{p}>0$, then $\lim \sup \sum_{k=0}^{\infty} a_{n k} s_{k} t_{k}\left(m_{k}\right)$ $>0$. This concludes the proof.

The proof actually shows

THEOREM. If $s \in L^{p}(A),\|s\|_{p}>0$ where $A$ is a nonnegative regular matrix and $p>1$, then for $p=2$ there exists a sequence $u \in L^{2}(A)$ such that $\|u\|_{2}$ 
$=1$ and $\lim \sup \sum a_{n k} s_{k} u_{k}=\|s\|_{2} ;$ for $p$ irrational there exists, for each positive $\delta$, a sequence $u \in L^{p^{\prime}}(A)$ such that $\|u\|_{p^{\prime}}=1$ and

$$
\lim \sup \sum a_{n k} s_{k} u_{k} \geqslant(1-\delta)\|s\|_{p} ;
$$

for $p$ rational, $p \neq 2$, there exists a sequence $u \in L^{p^{\prime}}(A)$ such that $\|u\|_{p^{\prime}}=1$ and $\lim \sup \sum a_{n k} s_{k} u_{k} \geqslant\|s\|_{p} \cos \pi / p^{\prime}$.

\section{REFERENCES}

1. J. R. Edwards and S. G. Wayment, A summability integral, J. Reine Angew Math. 255 (1972), 85-93. MR 46 \#4050.

2. L. Gillman and M. Jerison, Rings of continuous functions, Univ. Ser. in Higher Math., Van Nostrand, Princeton, N. J., 1960. MR 22 \#6994.

School of Mathematics, University of Minnesota, Minneapolis, Minnesota 55455 